\title{
IDEAÇÃO SUICIDA E TENTATIVA DE SUICÍDIO ENTRE PESSOAS EM TRATAMENTO PSIQUIÁTRICO ${ }^{1}$
}

\author{
SUICIDAL IDEATION AND SUICIDE ATTEMPT AMONG PEOPLE IN PSYCHIATRIC TREATMENT \\ IDEACIÓN SUICIDA E INTENTO DE SUICIDIO ENTRE PERSONAS EN TRATAMIENTO \\ PSIQUIÁTRICO
}

\author{
Nadja Cristiane Lappann Botti \\ Aline Conceição Silva* \\ Luiza Cantão ${ }^{* * *}$ \\ Thais Gonçalves Dias ${ }^{* * * *}$ \\ Ramon Azevedo Silva de Castro ${ }^{* * * *}$ \\ Júlia Esteves de Assunção ${ }^{* * * * *}$
}

\begin{abstract}
RESUMO
Este estudo investigou a relação entre ideação suicida, tentativa de suicídio e características psicossociais e psiquiátricas de pacientes com transtornos psiquiátricos. Realizado estudo quantitativo por meio da análise documental de prontuários de um centro de atenção psicossocial de uma cidade do interior do Sudeste do Brasil. Entre os 410 prontuários analisados, foi encontrado $24,4 \%$ com registro de ideação suicida e 22,9\% com tentativa de suicídio. Entre os pacientes com ideação suicida e tentativa de suicídio, verifica-se que a maioria são homens, adultos, sem vínculo matrimonial, com baixa escolaridade, inatividade laboral e apresentam os transtornos do humor como diagnóstico principal e comorbidade psiquiátrica de maior frequência. Identificou-se associação de ideação suicida e tentativa de suicídio com comorbidade psiquiátrica e tentativa de suicídio com história de experiência traumática. Importante realizar avaliação do risco dos pacientes com história de comportamento suicida para melhor manejo e prevenção do suicídio.
\end{abstract}

Palavras-chave: Transtornos mentais. Serviços de saúde mental. Tentativa de suicídio. Ideação suicida.

Texto recebido em 5 de dezembro de 2016 e aprovado para publicação em 26 de setembro de 2017.

1 Trabalho financiado pela Fundação de Amparo à Pesquisa de Minas Gerais (Fapemig), processo APQ-01641-14.

"Doutora pela Universidade de São Paulo (USP), professora Adjunta da Universidade Federal de São João del-Rei (UFSJ), psicóloga.E-mail: nadjaclb@terra.com.br.

${ }^{* *}$ Mestra pela UFSJ, enfermeira. E-mail: csilvaaline@hotmail.com.

*** Mestra pela UFSJ, enfermeira. E-mail: lulucantao@hotmail.com.

${ }^{* \ldots *}$ Especialista em Atenção Básica/Saúde da Família pela UFSJ, enfermeira. E-mail: thaisgdias27@gmail.com.

***** Mestrando pela USP/EERP, enfermeiro. E-mail: ramonazevedo_silva@hotmail.com.

****** Graduanda em Enfermagem pela UFSJ. E-mail: juuhesteves@hotmail.com. 


\begin{abstract}
This study investigated the relationship between suicidal ideation, suicide attempt and psychosocial and psychiatric characteristics of patients with psychiatric disorders. A quantitative study was carried out based on the documentary analysis of records at a psychosocial care center of a town in the southeast of Brazil. Among the 410 medical records analyzed, $24.4 \%$ were found to have suicidal ideation and $22.9 \%$ referred to suicide attempts. Among the patients with suicidal ideation and suicide attempt, it is verified that the majority are men, adults, without marital bond, with low educational level, work inactivity, and present mood disorders as the main diagnosis and psychiatric comorbidity of higher frequency. It was identified association of suicidal ideation and suicide attempt with psychiatric comorbidity and suicide attempt with history of traumatic experience. Important perform risk assessment of patients with a history of suicidal behavior to better management and suicide prevention.
\end{abstract}

Keywords: Mental disorders. Mental health services. Suicide attempt. Suicidal ideation.

\title{
RESUMEN
}

Este estudio investigó la relación entre ideación suicida, intento de suicidio y las características psicosociales y psiquiátricas de los pacientes con trastornos psiquiátricos. Estudio cuantitativo realizado por análisis documental de los expedientes de un centro de atención psicosocial de una ciudad en el sureste de Brasil. Entre los 410 registros de pacientes analizados se encontró $24,4 \%$ con registro de ideación suicida y $22,9 \%$ con intento de suicidio. Entre los pacientes con ideación suicida e intento de suicidio, se encontró que la mayoría son hombres, adultos, sin vínculo matrimonial, con bajo nivel de educación, inactividad laboral, y tienen los trastornos del ánimo como el diagnóstico primario y comorbilidad psiquiátrica más frecuente. Se identificó asociación de ideación suicida e intento de suicidio con comorbilidad psiquiátrica e intento de suicidio con historia de experiencia traumática. Importante realizar la evaluación del riesgo de los pacientes con antecedentes de comportamiento suicida para una mejor gestión y prevención del suicidio.

Palabras clave: Trastornos mentales. Servicios de salud mental. Intento de suicidio. Ideación suicida. 


\section{INTRODUÇÃO}

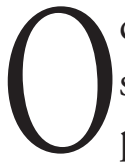

comportamento suicida caracteriza-se como importante problema de saúde pública. A tentativa de suicídio é um dos principais fatores de risco para o suicídio, sendo que até $50 \%$ das pessoas que cometeram suicídio apresentam, ao menos, uma tentativa prévia (Thornicrof, 2013) e 10\% das pessoas com tentativas morrem por suicídio num período de dez anos (Botega, 2012). Vidal, Gontijo e Lima (2013), em estudo realizado com 807 pessoas, no interior do Sudeste brasileiro, constataram que 60\% dos suicídios ocorreram após um ano da tentativa de suicídio e 90\% dos suicídios ocorreram no período de 24 meses após a tentativa. Ainda se verifica que, em 90\% dos suicídios, havia a presença de transtorno psiquiátrico, sendo que mais de dois terços não estavam realizando tratamento quando morreram (Vidal, Gontijo, \& Lima, 2013).

A tentativa de suicídio e suicídio consumado em pacientes psiquiátricos é maior do que na população em geral (Pires et al., 2015). A maioria dos pacientes que cometem suicídio apresenta transtorno psiquiátrico, principalmente depressão unipolar, transtorno afetivo bipolar, transtorno por uso de substâncias ou esquizofrenia (Teng \& Pampanelli, 2015). Nessa direção, Klonsky, May e Saffer (2016) enfatizam importante associação entre transtornos psiquiátricos e tentativas de suicídio devido ao fato de que vários transtornos contribuem para o desenvolvimento da ideação suicida.

Pacientes psiquiátricas com tentativa de suicídio, em geral, apresentam transtorno de adaptação ou transtorno de ajustamento e diferença na escolha do método de acordo com a faixa etária ou estado civil (Shakeri et al., 2015). Estudo brasileiro com pacientes com transtorno de ansiedade generalizada (TAG) com risco de suicídio encontra a depressão maior como principal comorbidade (Vasconcelos, Lôbo, \& Melo Neto, 2015).

A ideação suicida, eventos estressores de vida recentes, psicopatologia e desesperança são considerados fatores de risco proximais e atuam como precipitantes do risco de suicídio (Turecki, Ernest, Jollant, Labonté, \& Mechawar, 2012). May e Klonsky (2016) apontam maior frequência de depressão e transtorno estresse pós-traumático entre as pessoas com ideação quando comparadas com as histórias de tentativa de suicídio. Os transtornos de ansiedade, de uso de drogas e história de abuso sexual foram mais elevados entre as pessoas com tentativa de suicídio, em comparação com as pessoas com ideação.

Estudo com 7.348 adultos dos Estados Unidos mostra que mais de 30\% dos que apresentaram ideação com plano e, ou, tentativa de suicídio não receberam tratamento antes ou depois do planejamento ou tentativa (Choi, Di Nitto, \& 
Marti, 2015). Entre 3.897 pacientes psiquiátricos coreanos, observa-se que 43\% morreram por suicídio durante o período de observação de 2,5 anos (Choi, Park, Yi, \& Hong, 2012). Os autores salientam a necessidade de estratégias de prevenção do suicídio para pacientes com tentativa de suicídio admitidos em serviços de pronto-socorro ou hospital psiquiátrico (Choi, Park, Yi, \& Hong, 2012).

Pires et al. (2015), em estudo do tipo caso-controle com sobreviventes de tentativa de suicídio por envenenamento, em uma emergência de hospital público de uma capital do Nordeste brasileiro, identificam como indicadores de risco estar em dependência financeira de terceiros, ter sofrido abuso sexual na infância, ideação suicida, histórico de transtorno mental na família, ter algum transtorno psiquiátrico e, principalmente, comorbidade psiquiátrica. No modelo de regressão, foi possível estimar uma chance de tentativa de suicídio de até $94,0 \%$ na presença conjunta de quatro fatores.

Estudo psicodinâmico acerca dos processos de pensamento, emoções, motivações e experiências subjacentes que acompanham a tentativa de suicídio mostra que as experiências subjetivas de solidão e dor psíquica encontram-se ligadas à desesperança, pessimismo e desânimo, além disso, a ilusão da morte pode representar um convite a tentativa de suicídio (Rendón-Quintero \& Rodríguez-Gómez, 2016).

Nesse contexto, é importante a realização de estudos sobre as variáveis que podem influenciar os comportamentos suicidas, de forma a tornar possível a intervenção preventiva em múltiplos contextos (Figel, Menegatti, \& Pinheiro, 2013). Assim, este estudo propõe a investigação da relação entre ideação suicida, tentativa de suicídio e características psicossociais e psiquiátricas de pacientes com transtornos psiquiátricos atendidos no Centro de Atenção Psicossocial de uma cidade do interior do Sudeste brasileiro.

\section{MÉTODO}

\subsection{Tipo de estudo}

Realizado estudo transversal, exploratório, descritivo, com abordagem quantitativa.

\subsection{Participantes}

Participaram da pesquisa 410 pacientes atendidos no Centro de Atenção Psicossocial de uma cidade do interior de Minas Gerais. O serviço caracteriza- 
se como CAPS III e atende a população de 220 mil habitantes. A amostra foi calculada tendo por base uma população de aproximadamente 16 mil usuários atendidos no serviço desde a sua inauguração, em 1997, até o ano de 2014, considerando-se um erro amostral de 5\% e um nível de significância de 5\%. A amostra aleatória simples refere-se a 410 pacientes adultos e com transtorno mental grave e persistente.

\subsection{Materiais}

A coleta de dados ocorreu por meio da análise documental dos prontuários do serviço arquivados na própria unidade. Os prontuários analisados foram de atendimentos realizados no serviço de 1997 até 2014. A leitura dos prontuários ocorreu no período de abril a dezembro de 2015. Os dados foram extraídos diretamente dos prontuários sendo coletadas informações sobre as seguintes variáveis: idade, sexo, estado civil, situação escolar e laboral, diagnóstico psiquiátrico e comorbidade psiquiátrica (CID 10), histórico de comportamento suicida (ideação, tentativa ou morte), histórico familiar e pessoal. Para a coleta de dados, foi usado formulário construído especificamente para a investigação científica, no qual as variáveis foram definidas com base na literatura (World Health Organization, 2014).

\subsection{Procedimento}

Após a aprovação do projeto pelo comitê de ética em pesquisa (CAAE: 41768415.1.0000.5545), seguiu-se a seleção dos prontuários para análise. A seleção foi definida por meio de sorteio de 65 caixas de prontuários, sendo retirado de cada caixa os dez primeiros prontuários de pacientes atendidos no serviço. Para análise quantitativa, utilizaram-se de estatísticas descritivas e inferenciais, sendo que, no último caso, foram utilizados os testes de $\mathrm{X}^{2}$ ou o teste exato de Fischer. O nível de significância adotado foi de 5\%. A análise estatística foi realizada por meio do pacote estatístico Statistical Package for the Social Sciences 21.

\section{RESULTADOS}

Os dados sociodemográficos são relativos aos encontrados na folha de admissão do prontuário dos 410 pacientes em tratamento psiquiátrico no Centro de Atenção Psicossocial III. Verifica-se que são em maioria do sexo masculino (51,5\%), adultos entre 19 e 59 anos (87,3\%), sem vínculo matrimonial (solteiro, 
separado e viúvo) (57,8\%), sem filhos (54,1\%), com baixa escolaridade $(\leq 8$ anos de estudo) $(71,8 \%)$ e inatividade laboral $(70,6 \%)$ (tabela 1$)$.

Tabela 1 - Distribuição dos dados sociodemográficos entre pacientes em tratamento psiquiátrico no Centro de Atenção Psicossocial III (1997-2014)

\begin{tabular}{cc|cc}
\hline \multicolumn{1}{c|}{ Dados sociodemográficos } & (n) & (\%) \\
\hline Sexo & Feminino & 199 & 48,5 \\
& Masculino & 211 & 51,5 \\
\hline \multirow{2}{*}{ Faixa etária } & $\leq 18$ anos & 13 & 3,2 \\
& $19-59$ anos & 358 & 87,3 \\
& $\geq 60$ anos & 39 & 9,5 \\
\hline Estado Civil & Solteiro & 158 & 41,5 \\
& Casado/União estável & 161 & 42,3 \\
& Separado/Viúvo & 62 & 16,3 \\
\hline Filhos & Sim & 188 & 45,9 \\
& Não & 222 & 54,1 \\
\hline Escolaridade & $\leq 8$ anos de estudo & 267 & 71,8 \\
& $>8$ anos de estudo & 105 & 28,2 \\
\hline Situação laboral & Ativo & 91 & 29,4 \\
& Inativo & 219 & 70,6 \\
\hline
\end{tabular}

Fonte: elaborado pelos autores.

Entre os 410 prontuários analisados, 24,4\% tinham registro de ideação suicida, e $22,9 \%$, de tentativa de suicídio. Entre os cem pacientes que apresentaram ideação, 30,0\% foram admitidos por esse motivo no serviço especializado de saúde mental; 50,0\% também apresentaram tentativa de suicídio; e 3,0\% tiveram morte registrada por suicídio. Dos 94 pacientes que apresentaram história de tentativa, verifica-se que 20,2\% foram motivo de admissão no serviço especializado de saúde mental; $53,2 \%$ também apresentam ideação suicida; e $3,2 \%$ tiveram morte registrada por suicídio. Ao fim, foram computadas 266 tentativas, com média igual a 2,8 $\pm 8,2$ tentativas de suicídio por cada indivíduo.

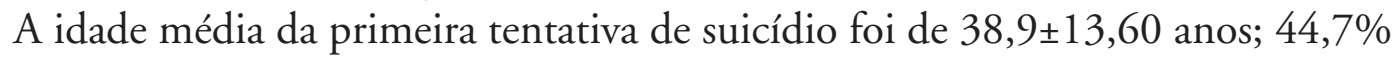
não apresentaram registro do meio de perpetração, sendo a autointoxicação o meio mais utilizado na primeira tentativa de suicídio $(23,4 \%)$. Esses dados são apresentados na tabela 2 . 
Tabela 2 - Meios de Perpetração (CID 10) da primeira tentativa de suicídio entre pacientes em tratamento psiquiátrico no Centro de Atenção Psicossocial III ( $n=94)$ (1997-2014)

\begin{tabular}{lcc}
\hline Meios de Perpetração (CID 10) da 1a tentativa de suicídio & (n) & (\%) \\
\hline $\begin{array}{l}\text { X61 - Autointoxicação por drogas anticonvulsivantes, sedativos, } \\
\text { hipnóticos, antiparkinsonianos e psicotrópicos }\end{array}$ & 20 & 21,3 \\
$\begin{array}{l}\text { X64 - Autointoxicação por outras drogas, medicamentos e } \\
\text { substâncias biológicas }\end{array}$ & 1 & 1,1 \\
X68 - Autointoxicação por pesticidas & 1 & 1,1 \\
X70 - Lesão autoprovocada por enforcamento, estrangulamento & 9 & 9,6 \\
e sufocação & 3 & 3,2 \\
X71 - Lesão por afogamento e submersão & 5 & 5,3 \\
X76 - Lesão pela fumaça, fogo e chamas & 6 & 6,4 \\
X78 - Lesão por objeto cortante ou penetrante & 5 & 5,3 \\
X80 - Lesão por precipitação de lugar elevado & 1 & 1,1 \\
X81 - Lesão por precipitação ou permanência diante de um & 1 & 1,1 \\
objeto em movimento & 1 & 44,7 \\
X82 - Lesão por impacto de veículo a motor & 42 & \\
Não especificado & & \\
\hline
\end{tabular}

\section{Fonte: elaborado pelos autores.}

Dos 94 pacientes em tratamento psiquiátrico no Centro de Atenção Psicossocial III com registro de tentativa de suicídio, verifica-se que são, em maioria, do sexo masculino $(53,2 \%)$, sem vínculo matrimonial (solteiro, separado e viúvo) $(62,3 \%)$, sem filhos $(58,5 \%)$, com baixa escolaridade ( $\leq 8$ anos de estudo) $(68,5 \%)$ e inatividade laboral $(72,2 \%)$, sendo características sociodemográficas similares dos pacientes em tratamento psiquiátrico no Centro de Atenção Psicossocial III. Em relação ao diagnóstico psiquiátrico, observa-se que o diagnóstico principal com maior frequência foram os transtornos do humor (F30-F39) $(29,8 \%$ ), seguido dos transtornos mentais e comportamentais devidos ao uso de substância psicoativa (F10-F19) (26,6\%) e da esquizofrenia, transtornos esquizotípicos e transtornos delirantes (F20-F29) (25,5\%). No tocante às comorbidades, verificase com maior os diagnósticos dos transtornos do humor (F30-F39) (29,5\%), seguido da esquizofrenia, transtornos esquizotípicos e transtornos delirantes (F20-F29) (18,2\%) e dos transtornos mentais e comportamentais devidos ao uso de substância psicoativa (F10-F19) (14,4\%) (tabela 3). 
Tabela 3 - Características sociodemográficas e psiquiátricas de pacientes com tentativa de suicídio em tratamento no Centro de Atenção Psicossocial III (1997-2014)

\begin{tabular}{|c|c|c|c|}
\hline & & (n) & (\%) \\
\hline \multirow{2}{*}{ Sexo } & Feminino & 44 & 46,8 \\
\hline & Masculino & 50 & 53,2 \\
\hline \multirow{3}{*}{ Faixa etária } & $\leq 18$ anos & 2 & 2,1 \\
\hline & $19-59$ anos & 87 & 92,6 \\
\hline & $\geq 60$ anos & 5 & 5,3 \\
\hline \multirow{3}{*}{ Estado Civil } & Solteiro & 42 & 46,7 \\
\hline & Casado/União estável & 34 & 37,8 \\
\hline & Separado/Viúvo & 14 & 15,6 \\
\hline \multirow{2}{*}{ Filhos } & Sim & 39 & 41,5 \\
\hline & Não & 55 & 58,5 \\
\hline \multirow{2}{*}{ Escolaridade } & $\leq 8$ anos de estudo & 61 & 68,5 \\
\hline & $>8$ anos de estudo & 28 & 31,5 \\
\hline \multirow{2}{*}{ Situação laboral } & Ativo & 20 & 27,8 \\
\hline & Inativo & 52 & 72,2 \\
\hline \multirow{5}{*}{ Diagnóstico psiquiátrico principal } & F00-F09 & 6 & 6,4 \\
\hline & F10-F19 & 25 & 26,6 \\
\hline & F20-F29 & 24 & 25,5 \\
\hline & F30-F39 & 28 & 29,8 \\
\hline & Outros & 11 & 11,7 \\
\hline
\end{tabular}

Fonte: elaborado pelos autores.

Quando observados os resultados do teste de inferências, observou-se que pacientes com história de comorbidade psiquiátrica apresentaram associação com ideação suicida e com tentativa de suicídio $(\mathrm{p}<0,001)$. Verifica-se ainda associação com a tentativa de suicídio a presença de história de experiência traumática como abuso físico ou sexual, acidente ou violência doméstica, violências, acidentes, perdas importantes, perdas ou separação dos pais e conflitos familiares $(\mathrm{p}<0,001)($ tabela 4$)$. 
Tabela 4 - Associação entre história psiquiátrica, ideação suicida e tentativa de suicídio de pacientes tratamento no Centro de Atenção Psicossocial III (1997-2014)

\begin{tabular}{|c|c|c|c|c|c|c|c|c|c|c|c|c|c|}
\hline \multirow{3}{*}{$\begin{array}{c}\text { História } \\
\text { psiquiátrica }\end{array}$} & & \multicolumn{6}{|c|}{ Ideação suicida } & \multicolumn{6}{|c|}{ Tentativa de suicídio } \\
\hline & & \multicolumn{2}{|l|}{ Sim } & \multicolumn{2}{|l|}{ Não } & \multirow[t]{2}{*}{$\chi^{2}$} & \multirow[t]{2}{*}{$\begin{array}{c}p \\
\text { valor }\end{array}$} & \multirow{2}{*}{$\begin{array}{l}\text { Sim } \\
\text { (n) }\end{array}$} & \multicolumn{3}{|c|}{ Não } & \multirow[t]{2}{*}{$\chi^{2}$} & \multirow[t]{2}{*}{$\begin{array}{c}p \\
\text { valor }\end{array}$} \\
\hline & & $(n)$ & $(\%)$ & (n) & $(\%)$ & & & & (\%) & (n) & (\%) & & \\
\hline \multirow{2}{*}{$\begin{array}{l}\text { Comorbidade } \\
\text { psiquiátrica }\end{array}$} & Sim & 68 & 68,0 & 145 & 46,8 & & & 70 & 74,5 & 143 & 45,3 & & \\
\hline & Não & 32 & 32,0 & 165 & 53,2 & 13,6 & 0,00 & 24 & 25,5 & 173 & 54,7 & 24,7 & 0,00 \\
\hline \multirow{2}{*}{$\begin{array}{c}\text { História } \\
\text { psiquiátrica } \\
\text { familiar }\end{array}$} & Sim & 62 & 62,0 & 177 & 57,1 & & & 58 & 61,7 & 181 & 57,3 & & \\
\hline & Não & 38 & 38,0 & 133 & 42,9 & 0,7 & 0,20 & 36 & 38,3 & 135 & 42,7 & 0,5 & 0,20 \\
\hline \multirow{2}{*}{$\begin{array}{l}\text { História de } \\
\text { experiência } \\
\text { traumática }\end{array}$} & Sim & 21 & 21,0 & 64 & 20,6 & & & 75 & 79,8 & 206 & 65,2 & & \\
\hline & Não & 79 & 79,0 & 246 & 79,4 & 0,0 & 0,50 & 19 & 20,2 & 110 & 34,8 & 7,1 & 0,00 \\
\hline
\end{tabular}

Fonte: elaborado pelos autores.

\section{DISCUSSÃO E CONCLUSÕES}

A amostra deste estudo apresenta alguns fatores de risco para o comportamento suicida quando se verifica que a maioria são adultos sem vínculo matrimonial, com baixa escolaridade e inatividade laboral. Estudo mostra que a maioria dos pacientes não eram casados entre os casos de suicídio consumado. Importante frisar que o vínculo marital não é um forte fator de proteção para tentativas de suicídio nos países em desenvolvimento. A qualidade da relação conjugal, afetividade, apoio da família estendida e capacidade de lidar com tensões relacionadas ao casamento e criação dos filhos são mais importantes que o estado civil propriamente dito (Radhakrishnan \& Andrade, 2012).

O desemprego pode elevar o risco de suicídio por meio de fatores como a pobreza, privação social, dificuldades domésticas e desesperança. Além disso, pacientes com transtornos psiquiátricos apresentam maior risco de suicídio como também maior propensão ao desemprego (Radhakrishnan \& Andrade, 2012). Entre adultos coreanos, verifica-se que a baixa escolaridade se encontra associada à tentativa de suicídio, que pode ser explicado pelo fato que, em geral, essas pessoas têm menor chance de competir por empregos e, portanto, adquirir maior renda e status social (Song \& Lee, 2016); como também menor resiliência e maior risco de desenvolver transtornos psiquiátricos. Estudo de coorte sueco mostra que pacientes com pensão por invalidez e com diagnóstico de transtornos depressivos, abuso de substâncias ou transtornos de personalidade apresentam 
maior risco para o comportamento suicida (Rahman, Alexanderson, Jokinen, \& Mittendorfer-Rutz, 2016).

A Organização Mundial de Saúde (OMS) aponta que os transtornos mentais graves estão associados a elevadas taxas de suicídio consumado, sendo 12 vezes maior entre pessoas com esquizofrenia em comparação com a população em geral. A OMS alerta que a presença de história de tentativas de suicídio, diagnóstico psiquiátrico de depressão ou abuso de álcool e, ou, outras drogas, não adesão ao tratamento psiquiátrico são fatores de risco para o suicídio entre pacientes com esquizofrenia e transtorno Organização Mundial de Saúde bipolar (World Health Organization, 2014).

É importante, portanto, a continuidade da observação do comportamento dos pacientes com história de ideação ou tentativa de suicídio após a alta do serviço de saúde mental para prevenir possível recidiva (Ferreira \& Gabarra, 2014). Nesse aspecto, é importante o profissional de saúde perceber que esses pacientes se encontram em situação de profundo sofrimento ao considerarem a tentativa de suicídio (Freitas \& Borges, 2014).

Kim et al. (2015), em estudo com 1.003 pacientes coreanos, apontam que, nas histórias de tentativa de suicídio planejadas, encontra-se maior frequência de pacientes mais velhos, divorciados, separados ou viúvos, com transtorno depressivo maior, transtornos por uso de substância e comorbidades médicas, além de maior disposição de autoacusação quando comparado com os pacientes com tentativa de suicídio impulsivo. Por outro lado, os conflitos interpessoais foram mais frequentes entre os pacientes com tentativa de suicídio impulsivo. Os pacientes com tentativa de suicídio planejado tinham menos tentativas de suicídio anteriores, entretanto eram tentativas mais graves. A presença de desesperança, testamento escrito e ideação suicida de natureza repetitiva, intensa e contínua foram preditivos de tentativa de suicídio planejado.

A ideação suicida aparece de acordo com os sentimentos, tristeza e problemas e situações vivenciados pela pessoa, desencadeando a tentativa de suicídio, neste caso pode-se entender o suicídio como a etapa final das ideaçôes suicidas (Zana \& Kovács, 2013). Tentativas de suicídio prévias constituem importante fator de risco para o suicídio e as tentativas sucessivas apresentam, em geral, métodos mais letais e consequentemente maior gravidade clínica (Botega, 2012). Pesquisa brasileira realizada em uma capital do Sul do País mostra que a totalidade apresentava ideação suicida antes de realizar o ato. A relação entre tentativa e ideação suicida se refere ao fato que geralmente as pessoas com ideação exibem ambiguidade de sentimentos, ora com vontade de viver, ora com desejo de morrer (Ferreira \& Gabarra, 2014). Szymanski, Aurelius, Szymanski e Lathrop 
(2016), em estudo com 342 casos de suicídio no México, apontam que a maioria apresentava ideação suicida, tentativas prévias de suicídio e nota suicida, e ainda afirmam que a doença psiquiátrica estava presente em $72 \%$ dos casos, sendo mais frequente a depressão, e que a maioria das mortes foram atribuídas à intoxicação por múltiplas drogas.

Ressalta-se que 44,7\% não apresentaram registro no prontuário do meio de perpetração, sendo que, dos meios registrados na primeira tentativa de suicídio, observa-se que o mais empregado foram as autointoxicações. A intoxicação exógena intencional ocorre com frequência na população adulta, sendo que, a cada três casos de intoxicação exógena acidentais, acontece um suicídio ou tentativa de suicídio (Campos, 2009).

A literatura mostra que pessoas com transtornos mentais graves apresentam maior risco de suicídio, como observado neste estudo. Estudo de coorte, durante dez anos, com pessoas com transtornos mentais graves mostra que o risco cumulativo de tentativa de suicídio foi de $26,3 \%$ para depressão maior, de 23,8\% para transtorno bipolar I e 13,1\% para a esquizofrenia (Shibre et al., 2014). Repetidas tentativas de suicídio encontram-se associadas com transtorno depressivo grave com ou sem sintomas psicóticos, transtorno bipolar tipo II, transtorno de uso de álcool e traços de transtorno de personalidade borderline (Aaltonen et al., 2016).

Pacientes com transtorno bipolar com história de tentativas de suicídio apresentam maiores escores de esquemas iniciais desadaptativos quando comparados àqueles sem história de tentativa, principalmente em relação ao isolamento social (crença de que é diferente dos outros, que não faz parte da sociedade e que está isolado do resto do mundo), independência (crença de que não é capaz de lidar com as responsabilidades do dia a dia com competência e independência) e noção de direitos (crença de que é superior às outras pessoas, com direitos e privilégios especiais e sem vinculação às regras de reciprocidade que orientam a interação social) (Nilsson, 2016).

A maioria dos comportamentos suicidas ocorrem no contexto de condiçóes psiquiátricas, principalmente da depressão e do transtorno bipolar (Antypa et al., 2016). Ainda nessas condiçôes psiquiátricas encontra-se o consumo de álcool como forte fator de risco proximal para tentativa de suicídio (Ghanbari, Malakouti, Nojomi, Leo, \& Saeed, 2016; Kennedy et al., 2015). Ainda em relação aos pacientes com diagnóstico de esquizofrenia, encontra-se como preditores de tentativas de suicídio a maior duração da internação, presença de sintomas depressivos e não adesão ao tratamento em fases iniciais do primeiro episódio de esquizofrenia (Togay, Noyan, Tasdelen, \& Ucok, 2015). 
Além das tentativas de suicídio, outros fatores são considerados como de risco para o suicídio, tais como perdas recentes, perdas de figuras parentais na infância, conflitos familiares, problemas financeiros, desemprego, morte e doença na família, personalidade com fortes traços de impulsividade e agressividade, doenças e acesso fácil a meios letais (Bertolote, 2012). Nesse sentido, identificouse associação com a tentativa de suicídio a presença de história de experiência traumática como abuso físico ou sexual, acidente ou violência doméstica, violências, acidentes, perdas importantes, perdas ou separação dos pais e conflitos.

Assim, observa-se, neste estudo, associação entre ter vivido experiências traumáticas com tentativa de suicídio entre os pacientes. Pode-se considerar que, a priori, a premissa que toda a vivência humana está integrada e, portanto, as experiências negativas continuam a ter influência na vida da pessoa. Estudos que exploram a relação entre exposição a experiências adversas e, ou, traumáticas ocorridas na infância e, ou, adolescência e o estado de saúde na vida adulta mostram que essa exposição está associada a consequências negativas para a pessoa em várias dimensões, como problemas físicos, psicológicos, cognitivos, comportamentais, sociais e emocionais (Pinto, Alves, \& Maia, 2015). Pode-se entender que os tipos de experiências traumáticas mais comuns, como abuso físico, emocional ou sexual e negligência emocional ou física causam forte estresse e sofrimento (Camblor et al., 2016).

Ressalta-se que grande parte dessas experiências ocorrem no domínio do ambiente familiar produzindo maior trauma nas vítimas. Dentro dos efeitos psicológicos produzidos pelas vivências traumáticas, encontram-se mudanças de personalidade e desenvolvimento emocional, insegurança, baixa autoestima e dificuldades nas relações sociais. Tais efeitos psicológicos levam, em geral, a pessoa a ter dificuldades para expressar sentimentos e reconhecer emoções negativas produzindo menor habilidade para reagir ao sofrimento (Camblor et al., 2016). Como as experiências traumáticas apresentam relação direta com ideação e tentativa de suicídio seria importante o desenvolvimento da capacidade de resiliência entre os pacientes com ajuda dos profissionais de saúde, amigos, familiares e a comunidade no qual está inserido (Forstadt, Cooper, \& Andrews, 2015).

Por fim, coloca-se como fatores limitantes na elaboração deste trabalho a qualidade da informação sobre a ocorrência do comportamento suicida devido à dificuldade de compreensão das informaçôes, escassez de dados nas evoluções e anotações da equipe multiprofissional e possibilidade do não registro da ocorrência de comportamento suicida. 
Ressalta-se que a qualidade dos registros nos prontuários também se configura como possíveis limitações no manejo do paciente com comportamento suicida, pelo fato de que a avaliação do risco também se baseia no registro de informações referentes aos fatores de risco e proteção, características do ato, precedentes pessoais ou familiares e identificação de redes de apoio social. Registros que, quando descritos nos prontuários, podem melhorar a assistência e avaliação do risco dos pacientes com comportamento suicida e operar na prevenção de uma morte evitável. 


\section{REFERENNCIAS}

Aaltonen K., Näätänen, P., Heikkinen, M., Koivisto, M., Baryshnikov, I., Karpov, B., .. . Isometsä, E. (2016). Differences and similarities of risk factors for suicidal ideation and attempts among patients with depressive or bipolar disorders. Journal of Affective Disorders, 15(193), 318-330.

Antypa, N., Souery, D., Tomasini, M., Albani, D., Fusco, F., Mendlewicz, J., Serretti, A. (2016). Clinical and genetic factors associated with suicide in mood disorder patients. European Archives of Psychiatry and Clinical Neurosciences, 266(2), 181-193.

Bertolote, J. M. (2012). O suicídio e sua prevenção. São Paulo: Unesp.

Botega, N. J. (2012). Prática psiquiátrica no hospital geral: interconsulta e emergência. Porto Alegre: Artmed.

Camblor, N., Pedrero, E. F., Paino, M., Álvarez, L. G., Ruiz, J. P. P., \& Giráldez, S. L. (2016). Evaluación de experiencias traumáticas tempranas en adultos. Papeles del Psicólogo, 37(1), 36-44.

Campos, I. (2009). Vidas interrompidas. Vitória: Departamento de Imprensa Oficial.

Choi, J., Park, S., Yi, K., \& Hong, H. (2012). Suicide mortality of suicide attempt patients discharged from emergency room, nonsuicidal psychiatric patients discharged from emergency room, admitted suicide attempt patients, and admitted nonsuicidal psychiatric patients. Archives of Suicide Research, 43, 235-43.

Choi, N. G., Di Nitto, D. M., \& Marti, C. N. (2015). Mental health treatment use and perceived treatment need among suicide planners and attempters in the United States: between and within group differences. Biomed Central Research Notes, 8, 305.

Ferreira, C. L. B., \& Gabarra, L. M. (2014). Pacientes em risco de suicídio: avaliação da ideação suicida e o atendimento psicológico. Unopar Científica Ciência Humanas e Educação, 16(2), 113-122.

Figel, F., Menegatti, C., \& Pinheiro, E. (2013). Tentativas de suicídio: uma análise de contingências. Estudos de Psicologia, 30(2), 211-218. 
Forstadt, L., Cooper, S., \& Andrews, M. (2015). Changing medicine and building community: maine's adverse childhood experiences momentum. Permanente Journal, 19(2), 92-95.

Freitas, A. P. A., \& Borges, L. M. (2014). Tentativas de suicídio e profissionais de saúde: significados possíveis. Estudos e Pesquisas em Psicologia, 14(2), 560-577.

Ghanbari, B., Malakouti, S. K., Nojomi, M., Leo, D., \& Saeed, K. (2016). Alcohol abuse and suicide attempt in iran: a case-crossover study. Global Journal of Health Science, 8(7), 58-67.

Kennedy, M. C., Marshall, B. D. L., Hayashi, K., Nguyen, P., Wood, E., \& Kerr, T. (2015). Heavy alcohol uses and suicidal behavior among people who use illicit drugs: a cohort study. Drug and Alcohol Dependence, 151, 272-277.

Kim, J., Lee, K. S., Kim, D. J., Hong, S. C., Choi, K. H., Oh, Y., . . Lee, K. U. (2015). Characteristic Risk factors associated with planned versus impulsive suicide attempters. Clinical Psychopharmacology and Neuroscience, 13(3), 308315.

Klonsky, E. D., May, A. M., \& Saffer, B. Y. (2016). Suicide, suicide attempts, and suicidal ideation. Annual Review of Clinical Psychology, 12, 307-330.

May, A. M., \& Klonsky, E. D. (2016). What distinguishes suicide attempters from suicide ideations?: a meta-analysis of potential factors. Clinical Psychology: Science and Practice, 23(1), 5-20.

Nilsson, K. K. (2016). Early maladaptive schemas in bipolar disorder patients with and without suicide attempts. Journal of Nervous and Mental Disease, 204(3), 236-239.

Pinto, V. C. P., Alves, J. F. C., \& Maia, A. C. (2015). Adversidade na infância prediz sintomas depressivos e tentativas de suicídio em mulheres adultas portuguesas. Estudos de Psicologia, 34(4), 617-625.

Pires, M. C. C., Raposo, M. C. F., Sougey, E. B., Bastos Filho, O. C., Silva, T. S., \& Passos, M. P. (2015). Indicadores de risco para tentativa de suicídio por envenenamento: um estudo caso-controle. Jornal Brasileiro de Psiquiatria, 64 (3), 193-199.

Radhakrishnan, R., \& Andrade, C. (2012). Suicide: an indianperspective. Indian Journal of Psychiatry, 54(4), 304-319. 
Rahman, S. G., Alexanderson, K., Jokinen, J., \& Mittendorfer-Rutz, E. (2016). Disability pension due to common mental disorders and subsequent suicidal behaviour: a population-based prospective cohort study. BMJ Open, 6(4), e010152.

Rendón-Quintero, E., \& Rodríguez-Gómez, R. (2016). Vivencias y experiencias de individuos con ideación e intento suicida. Revista Colombiana de Psiquiatría, 45(2), 92-100.

Shakeri, J., Farnia, V., Abdoli, N., Akrami, M. R., Arman, F., \& Shakeri, H. (2015). The risk of repetition of attempted suicide among iranian women with psychiatric disorders as quantified by the suicide behaviors questionnaire. Oman Medical Journal, 30(3), 173-180.

Shibre, T., Hanlon, C., Medhin, G., Alem, A., Kebede, D., Teferra, S., . . ., Fekadu, A. (2014). Suicide and suicide attempts in people with severe mental disorders in Butajira, Ethiopia: 10-year follow-up of a population-based cohort. Biomedical Central Psychiatry, 14, 150.

Song, H. B., \& Lee, S. A. (2016). Socioeconomic and lifestyle factors as risks for suicidal behavior among Korean adults. Journal of Affective Disorders, 197, 21-28.

Szymanski, L. J., Aurelius, M. B., Szymanski, S. A., \& Lathrop, S. L. (2016). Suicidal drug overdoses in New Mexico: a 5-year retrospective review. Journal of Forensic Sciences, 61(3), 661-5.

Teng, C. T., \& Pampanelli, M. B. (2015). O suicídio no contexto psiquiátrico. Revista Brasileira de Psicologia, 2(1), 41-51.

Thornicrof, G. (2013). Premature death among people with mental illness. British Medical Journal, 3(346), f3423.

Togay, B., Noyan, H., Tasdelen, R., \& Ucok, A. (2015). Clinical variables associated with suicide attempts in schizophrenia before and after the first episode. Psychiatry Research, 229(1-2), 252-256.

Turecki, G., Ernest, C., Jollant, F., Labonté, B., \& Mechawar, N. (2012). The development origins of suicidal behavior. Trends in Neurosciences, 35(1), 1423. 
Vasconcelos, J. R. O., Lôbo, A.P. S., \& Melo Neto, V. L. (2015). Risco de suicídio e comorbidades psiquiátricas no transtorno de ansiedade generalizada. Jornal Brasileiro de Psiquiatria, 64(4), 259-265.

Vidal, C. E. L., Gontijo, E. C. D. M., \& Lima, L. A. (2013). Tentativas de suicídio: fatores prognósticos e estimativa do excesso de mortalidade. Caderno de Saúde Pública, 29(1), 175-187.

World Health Organization (2014). Preventing suicide: a global imperative. Geneva: World Health Organization.

Zana, A. R. O., \& Kovács, M. J. (2013). O psicólogo e o atendimento a pacientes com ideação ou tentativa de suicídio. Estudos e Pesquisas em Psicologia, 13(3), 897-921. 